\title{
Viability of Glioblastoma Cells and Fibroblasts in the Presence of Imidazol-Containing Compounds
}

\author{
Elisabeth Christiane Seidel ${ }^{1,4}$, Claudia Birkemeyer ${ }^{2}$, Rainer Baran-Schmidt ${ }^{1}$, Jürgen Meixensberger ${ }^{1}$, Henry Op- \\ permann ${ }^{1,3,+}$ and Frank Gaunitz ${ }^{1,+, *}$
}

\author{
Klinik und Poliklinik für Neurochirurgie, Universitätsklinikum Leipzig AöR, 04103 Leipzig, Germany \\ Institut für Analytische Chemie, Universität Leipzig, 04103 Leipzig, Germany \\ Institut für Humangenetik, Universitätsklinikum Leipzig AöR, 04103 Leipzig, Germany \\ 4 Klinik und Poliklinik für Neurologie, Universitätsklinikum Leipzig AöR, 04103 Leipzig, Germany \\ + These authors contributed equally \\ * Correspondence: Frank.Gaunitz@medizin.uni-leipzig.de; Tel. +49 341 97-17953; Fax.: +49 341 97- \\ 17509
}

\begin{abstract}
The naturally occurring dipeptide carnosine ( $\beta$-alanyl-L-histidine) specifically attenuates tumor growth. Here, we asked whether other small imidazole-containing compounds also affect viability of tumor cells without affecting non-malignant cells, and whether formation of histamine is involved. Patient-derived fibroblasts and glioblastoma cells were treated with carnosine, L-alanyl-L-histidine (LA-LH), ß-alanyl-L-alanine, L-histidine, histamine, imidazole, $\beta$-alanine and L-alanine. Cell viability was assessed by cell-based assays and microscopy. The intracellular release of L-histidine and formation of histamine was investigated by High Performance Liquid Chromatography coupled Mass Spectrometry. Whereas carnosine and LA-LH inhibited tumor cell growth with minor effects on fibroblasts, L-histidine, histamine and imidazole affected viability in both cell types. Compounds without imidazole moiety did not diminish viability. In the presence of LA-LH but not in the presence of carnosine a significant rise of intracellular amounts of histidine was detected in all cells. Formation of histamine was not detectable in the presence of carnosine, LA-LH or histidine. In conclusion, the imidazole moiety of carnosine contributes to its anti-neoplastic effect, which is also seen in the presence of histidine and LA-LH. Despite histamine had a strong effect on cell viability, formation of histamine is not responsible for the effects on cell viability of carnosine, LA-LH and histidine.
\end{abstract}

Keywords: carnosine; glioblastoma; fibroblasts; imidazole-containing compounds; cell viability; high performance liquid chromatography coupled mass spectrometry

\section{Introduction}

With 3.23 new cases per 100,000 inhabitants in the United States, glioblastoma (GBM) is the most frequent malignant tumor of the human brain [1]. GBM is an astrocytic tumor and is classified according to the World Health Organization (WHO) with the highest WHO grade IV. Despite the best possible treatment consisting of maximal safe resection of the tumor, radiotherapy and adjuvant chemotherapy with temozolomide, the 5-year overall survival of GBM patients is only 7.2\% [1]. Furthermore, the effectivity of this therapy is highly dependent on genetic properties of the tumor, namely the methylation status of the O-6-methylguanine-DNA Methyltransferase (MGMT) promotor [2]. In view of the poor prognosis and missing alternatives to the standard therapy there is ongoing research for new treatment strategies and drugs that could improve the corresponding outcome.

In recent years, we and others demonstrated that the naturally occurring dipeptide L-carnosine ( $\beta$-alanyl-L-histidine; Note: throughout the further text "carnosine" refers to "L-carnosine"), which was originally discovered more than 120 years ago [3], may be a potential anti-neoplastic drug for different types of cancer in general and also for glioblastoma in particular (for review see [4,5]). As carnosine is rapidly degraded in human 
plasma due to the presence of serum carnosinase it has long been thought that its use as a systemically administered drug may be limited. Although, there is now evidence, that carnosine can escape from degradation by uptake into erythrocytes [6] other compounds with higher stability might be considered as alternatives. Therefore, it is reasonable to better understand carnosine's mode of action on tumor cell viability which also requires an understanding of the bioactive characteristics of the molecule. In view of the observation that L-histidine is able to mimic carnosine's anti-neoplastic effect [7], we asked whether other small imidazole containing compounds are also able to mimic carnosine's effect, whether the $\beta$-alanyl moiety is required and whether the effects of other compounds are indeed comparable to that of carnosine. In addition, we also wanted to know whether the formation of histamine from L-histidine is involved in the anti-neoplastic effect.

\section{Results}

Viability of glioblastoma cells and patient-derived fibroblasts in the presence of imidazole-containing compounds

In a first series of experiments, we investigated the effect of imidazole-containing compounds on cell viability using five patient-derived fibroblast cell cultures (13/16, 90/15, 69/15, 60/15, 52/15) and four glioblastoma cell lines (U87, T98G, U87, G55T2). The cells were incubated for 48 hours in the presence of carnosine, L-alanyl-L-histidine (LA-LH), $\beta$-alanyl-L-alanyl ( $\beta$ A-LA), L-histidine, histamine, imidazole, L-alanine and $\beta$-alanine (all $50 \mathrm{mM}$ ), and cell viability was determined measuring the amount of ATP in cell lysates and dehydrogenase (DH) activity in living cells. Data was compared to untreated control cells. The results of experiments with fibroblasts from culture 52/15 and cells from the glioblastoma cell line U343 are presented in Figure 1 (all other experiments are presented in detail in Supplement 1).

In summary, as revealed by both assays, carnosine significantly reduced viability in all glioblastoma cells to at least $80 \%$ (with the exception of measuring DH in T98G). As shown in Table 1, fibroblasts also responded with a small reduction of viability in the presence of carnosine in almost all cells (maximal reduction to 90\%), which was in all cases lower than that observed in GBM cell lines. Using LA-LH, we could detect also a significant reduction of viability in glioblastoma cells as determined by both assays, whereas the viability of fibroblasts was not affected by the compound. Overall carnosine and LA-LH exerted a comparable impact on glioblastoma cells with differences regarding the various cell lines. Histidine reduced glioblastoma cell viability stronger than carnosine and LALH and significantly affected viability of fibroblasts. In contrast, the non-Imidazolyl containing dipeptide $\beta A$-LA did neither reduce GBM nor fibroblast viability. Histamine strongly reduced fibroblast viability and glioblastoma cell viability without a significant difference between both cell types. Comparing the effect of histamine with histidine, a significantly stronger reduction by histamine was detected in fibroblasts but not in glioblastoma cells. Imidazole also strongly reduced the viability of fibroblasts and glioblastoma cells without discriminating between the cell types. In comparison to imidazole, histamine stronger reduced cell viability of fibroblasts and exerted a comparable effect on glioblastoma cells. 

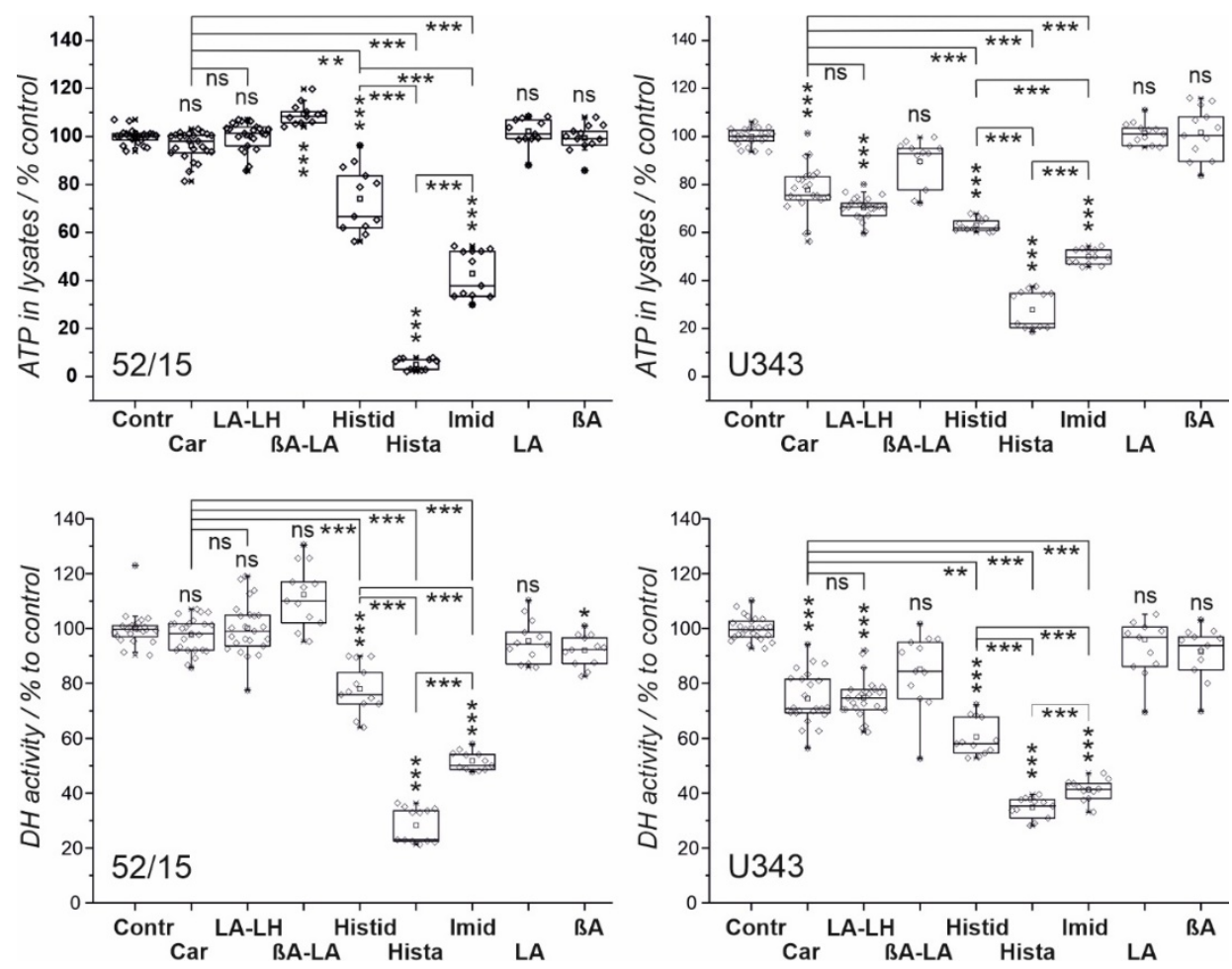

Figure 1. Viability of fibroblasts from culture 52/15 (left) and glioblastoma cells from the line U343 (right) after treatment with different compounds. Cells were treated for 48 hours with carnosine (Car), L-alanyl-L-histidine (LA-LH), $\beta$-alanylL-alanine ( $\beta$ A-LA), L-histidine (Histid), histamine (Hista), imidazole (Imid), L-alanine (LA) and $\beta$-alanine ( $\beta$ A) (all $50 \mathrm{mM})$ or vehicle control (Contr) for 48 hours. Cell viability was measured by determining ATP in cell lysates (upper panels) and dehydrogenase activity $(\mathrm{DH})$ in living cells (lower panels). Results are presented as box-plots. Statistical analysis was performed using a one-way ANOVA. The level of significance between different compounds is indicated by horizontal lines and compared to Contr above the boxes: * $\mathrm{p}<0.05 ;{ }^{* *}: \mathrm{p}<0.005 ;{ }^{* * *}: \mathrm{p}<0.0005$; ns: not significant.

The summary of all data obtained is shown in Table 1.

Table 1. Effect of different compounds on viability of fibroblasts and glioblastoma cells. The reduction of ATP in cell lysates and dehydrogenase activity $(\mathrm{DH})$ in living cells is color-indicated compared to untreated control cells after 48-hour exposure to the compounds. Imidazole-containing compounds are shown in bold. Note: only statistically significant effects are indicated, and white fields indicate no significance.

\begin{tabular}{|c|c|c|c|c|c|c|c|c|c|c|c|c|c|c|c|c|c|c|}
\hline & \multicolumn{10}{|c|}{ Fibroblasts } & \multicolumn{8}{|c|}{ Glioblastoma cells } \\
\hline \multirow{2}{*}{\begin{tabular}{|l|} 
Culture \\
Assay \\
\end{tabular}} & \multicolumn{2}{|c|}{$13 / 16$} & \multicolumn{2}{|c|}{$90 / 15$} & \multicolumn{2}{|c|}{$69 / 15$} & \multicolumn{2}{|c|}{$60 / 15$} & \multicolumn{2}{|c|}{$52 / 15$} & \multicolumn{2}{|c|}{ U343 } & \multicolumn{2}{|c|}{ T98G } & \multicolumn{2}{|c|}{ U87 } & \multicolumn{2}{|c|}{ G55T2 } \\
\hline & ATP & $\mathrm{DH}$ & ATP & $\mathrm{DH}$ & ATP & $\mathrm{DH}$ & ATP & $\mathrm{DH}$ & ATP & $\mathrm{DH}$ & ATP & $\mathrm{DH}$ & ATP & $\mathrm{DH}$ & ATP & $\mathrm{DH}$ & ATP & $\mathrm{DH}$ \\
\hline Carnosine & & & & & & & & & & & & & & & & & & \\
\hline LA-LH & & & & & & & & & & & & & & & & & & \\
\hline Histidine & & & & & & & & & & & & & & & & & & \\
\hline Imidazole & & & & & & & & & & & & & & & & & & \\
\hline Histamin & & & & & & & & & & & & & & & & & & \\
\hline$\beta$ Ala-L-Alc & & & & & & & & & & & & & & & & & & \\
\hline L-Ala & & & & & & & & & & & & & & & & & & \\
\hline$\beta$-Ala & & & & & & & & & & & & & & & & & & \\
\hline
\end{tabular}


Necrosis and morphological changes of GBM cells and patient-derived fibroblasts after treatment with different imidazole-containing compounds

In the preceding section, we analyzed viability of GBM cells and fibroblasts by measuring ATP in cell lysates and dehydrogenase activity in living cells after treatment with different compounds. These experiments indicated that carnosine and LA-LH reduced the investigated parameters clearly more in GBM cells than in in fibroblasts. Whereas histidine and imidazole also appeared to affect GBM cells more severely than fibroblasts, histamine affected fibroblasts and tumor cells similarly. Nonetheless, only carnosine and LALH appeared to have no effect on the viability of fibroblasts, although they obviously inhibited the production of ATP and dehydrogenase activity in GBM cells in general to below of $80 \%$.

In order, to get a more detailed picture on the effect of the compounds on GBM cells and fibroblasts, we investigated next morphological changes after treatment with the selected compounds, and we determined cell numbers and necrotic cells by propidium iodide staining. In addition, we also stained living cells using Calcein-AM. For this experiment, cells from the four glioblastoma cell lines G55T2, T98G, U87 and U343 and from fibroblast cultures $(90 / 15,69 / 15,60 / 15$ and 52/15) were exposed to our compounds (each $50 \mathrm{mM}$ ) for 48 hours. Then, staining with Hoechst 33343, Calcein-AM and propidium iodide was performed and the effects were monitored by phase contrast and fluorescence microscopy. Figure 2 presents the result of the experiment and its analysis along with representative pictures of cells from the line U343 and from fibroblast culture 52/15. 

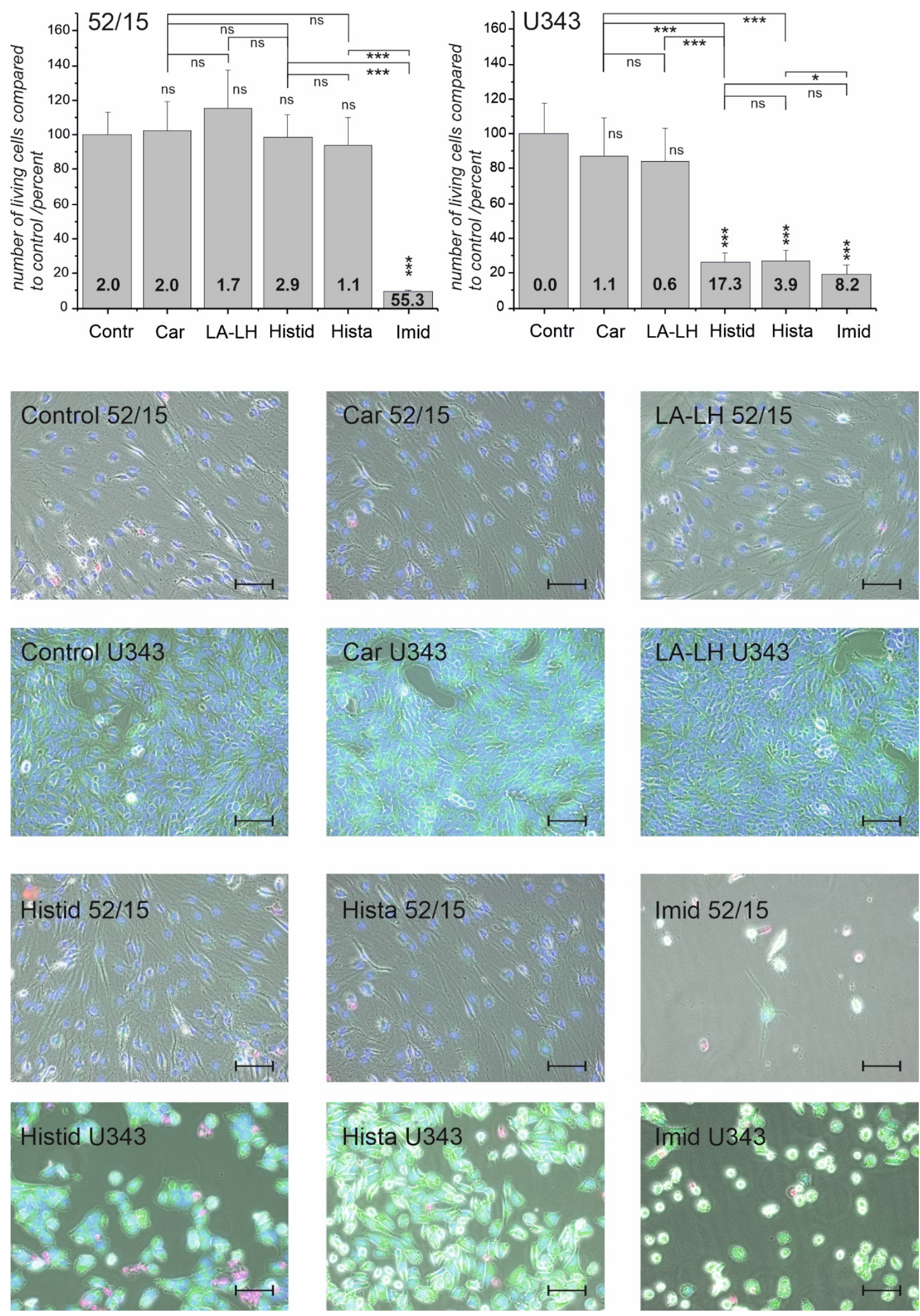

Figure 2. Microscopic analysis of fibroblasts (52/15) and glioblastoma cells (U343) in the presence of different compounds. Cells were treated for 48 hours with carnosine (Car), L-alanyl-L-histidine (LA-LH), $\beta$-alanyl-L-alanine ( $\beta$ A-LA), L-histidine (Histid), histamine (Hista), imidazole (Imid), L-alanine (LA) and $\beta$-alanine ( $\beta \mathrm{A})$ (all $50 \mathrm{mM}$ ) or vehicle control 
(Contr) for 48 hours. Then, microscopic images after staining with Hoechst 33343 (nuclei, blue), propidium iodide (dead cells, red) and Calcein-AM (living cells, green) were compared (representative images are presented as overlays in the lower panels that also include an image obtained by phase contrast). In order to determine the number of living cells using ImageJ, the total number of nuclei was determined, subtracting nuclei of dead cells (bars in upper panel; Note: determination of living cells from images of cells positive for Calcein-AM staining was not performed because of high errors due to technical reasons). The ratio of dead cells to living cells is presented by bold numbers in the bars in the upper panel. Statistical analysis was performed using a one-way ANOVA. The level of significance between different compounds is indicated by horizontal lines and compared to Contr above the bars: ${ }^{*}: \mathrm{p}<0.05 ;{ }^{* *}: \mathrm{p}<0.005 ;{ }^{* * *}$ : $<<0.0005$; ns: not significant. (Size bars: $100 \mu \mathrm{m})$.

Pictures from experiments with other cell lines and fibroblast cultures are presented in Supplement 2. In addition, a summary of the observations from all cells is given in Table 2.

\begin{tabular}{|c|c|c|c|c|c|c|c|c|c|c|c|c|c|c|c|c|}
\hline \multirow[b]{2}{*}{ culture } & \multicolumn{8}{|c|}{ Fibroblasts } & \multicolumn{8}{|c|}{ Glioblastoma } \\
\hline & $90 / 15$ & & $69 / 15$ & & $60 / 15$ & & $52 / 15$ & & U343 & & T98G & & G55T2 & & U87 & \\
\hline & $\%$ & ratio & $\%$ & ratio & $\%$ & ratio & $\%$ & ratio & $\%$ & ratio & $\%$ & ratio & $\%$ & ratio & $\%$ & ratio \\
\hline \multicolumn{17}{|l|}{ Car } \\
\hline \multicolumn{17}{|l|}{ LA-LH } \\
\hline \multicolumn{17}{|l|}{ Histid } \\
\hline \multicolumn{17}{|l|}{ Imid } \\
\hline \multicolumn{17}{|l|}{ Hista } \\
\hline living c & ells to $\mathrm{cc}$ & ontrol (9 & & & $<100-90$ & $<<90-80$ & $<80-$ & & & $<60-50$ & $<50-40$ & $<40-3$ & & 20 & & $<10$ \\
\hline ratio de & $\mathrm{ad} /$ livin & & & & $\leq 0.5$ & $>0.5-1$ & $>1-2$ & $>2$ & & $>4-8$ & $>8-16$ & $>16-$ & $32>32$ & $-64>$ & & \\
\hline
\end{tabular}

Table 2. Comparison of living cells and the ratio between dead and living cells in fibroblasts and glioblastoma cell cultures under the influence of different compounds. The total number of living cells in four fibroblast cultures and four glioblastoma cell lines after treatment with different compounds compared to untreated control cells (in \%) as well as the ratio between dead cells and living cells is color-indicated.

In summary, the strongest reduction of the number of living cells was achieved in the presence of imidazole, which did not significantly discriminate between fibroblasts or glioblastoma cells, indicating a generally high toxicity of this compound for both cell types. In some cases (e.g., U87), loss of cells was that high, that the calculation of the ratio between dead and living cells was not useful ("nd" in Table 2). Histidine and histamine on the other hand affected fibroblasts and glioblastoma cells significantly different with much more dead cells in the latter ones. Compared to histidine and histamine, carnosine and LA-LH seem to be less toxic but, in most cases, exhibited a stronger effect on glioblastoma cells than on fibroblasts. Comparing the cell counts presented in Table 2 to the effects on physiological parameters (Table 1) it seems to be likely, that the effects of the different compounds on cells may differ with regard to their mechanisms of action on energy metabolism.

\section{The release of L-histidine from L-alanyl-L-histidine does not result in formation of histamine}

Next, we investigated whether different amounts of L-histidine are released from carnosine and LA-LH and whether significant intracellular amounts of histamine can be formed from intracellular L-histidine. Figure 3a presents the intracellular amounts of Lhistidine when cells are incubated in the presence of carnosine or LA-LH, respectively (both $50 \mathrm{mM}$ ) as determined in fibroblast cell cultures $(\mathrm{n}=5)$ and glioblastoma cell lines $(n=4)$. As can be seen, no significant increase of intracellular L-histidine compared to untreated control cells was observed in cells incubated in the presence of carnosine. In contrast, incubation in the presence of LA-LH resulted in a significant increase of intracellular L-histidine. In order to detect a possible formation of histamine, we incubated cells from the GBM line T98G directly in the presence of L-histidine $(25 \mathrm{mM})$ and histamine $(25 \mu \mathrm{M})$ 
and determined the amount of both compounds in the cells. As can be seen in Figure $3 \mathrm{~b}$ we could not detect histamine in cells incubated in the presence of L-histidine, although we observed a steep raise in the intracellular amount of histamine when added to the medium. In addition, we did not detect histamine or its degradation products in cells exposed to carnosine, LA-LH or to L-histidine, whereas we observed formation of $\mathrm{N}$ Methylhistamine in cells cultivated in the presence of histamine.
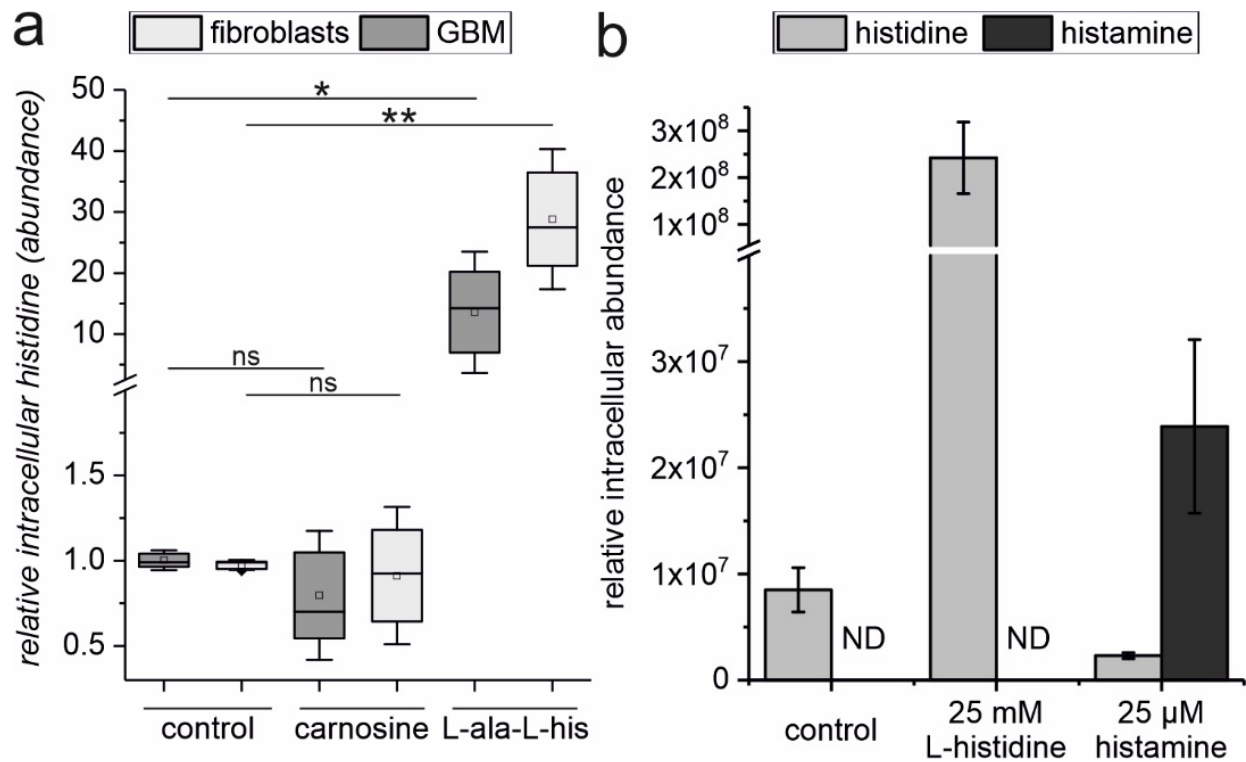

Figure 3. Release of histidine from carnosine and LA-LH and formation of histamine from histidine in fibroblasts and glioblastoma cells. a) Four different glioblastoma cell lines and five different fibroblast cultures were treated with carnosine or L-alanyl-L-histidine (LA-LH) (both $50 \mathrm{mM}$ ) or vehicle control for 48 hours. Afterwards, metabolites were extracted and intracellular histidine was determined by LC-MS. Results are presented as box-plots that were obtained from the median of the replicates of each cell culture. b) T98G cells were treated with $25 \mathrm{mM}$ L-histidine, $25 \mu \mathrm{M}$ histamine or vehicle control for 48 hours. Afterwards, metabolites were extracted and intracellular histamine and L-histidine were determined by LC-MS. Statistical analysis was performed using a one-way ANOVA with the Games-Howell post hoc test. The level of significance is indicated as: ${ }^{*}: \mathrm{p}<0.05 ;{ }^{* *}: \mathrm{p}<0.005$; not significant (ns): $\mathrm{p}>0.05$. ND: not detected.

\section{Discussion}

Several decades ago the anti-neoplastic effect of carnosin was first described in vivo by Nagai and Suda [8]. Later, this observation was confirmed in vivo and in vitro by several groups and different types of cancer such as gastric carcinoma [9], colon carcinoma [10], cervical carcinoma [11] and glioblastoma [12]. Just recently, we demonstrated that L-histidine, one amino acid of the dipeptide, reduces GBM cell viability even more potently than carnosine [13]. Therefore, we wondered whether L-histidine itself or other histidinecontaining compounds would also discriminate between malignant GBM cells and nonmalignant fibroblasts as demonstrated for carnosine [14,15]. Here, we observed that carnosine significantly reduced cell viability in GBM cells, whereas there was no significant effect observed in fibroblasts with regard to the amount of ATP in cell lysates and only a small but significant effect with regard to dehydrogenase activity. A comparable discrimination between tumor and non-tumor cells was also observed after application of LALH. Although L-histidine did significantly reduce viability of fibroblasts, the effect was stronger in GBM cells but significantly different between GBM cells and fibroblasts only with regard to dehydrogenase activity.

As L-histidine also has anti-neoplastic effects in GBM cells, this observation raises the question whether L-histidine could be used as a therapeutic agent instead of carnosine. First of all, at the concentrations employed in our experiments $(50 \mathrm{mM})$ the amino acid obviously also affects fibroblasts. Comparable observations have been made by Rauen et al. who detected that in cultivated liver cells, L-histidine at a concentration of $76 \mathrm{mM}$ had 
a 2.5 higher toxicity compared to carnosine at a concentration of $198 \mathrm{mM}$ [16]. As L-histidine is a proteinogenic amino acid, it could also be assumed that orally ingested L-histidine is taken up by other cells and may not reach the tumor. Orally applied carnosine on the other hand is rapidly degraded by serum carnosinase [17] and may therefore be limited in delivering its histidine moiety to cancer cells. In this regard, it has to be noted that we just recently demonstrated that carnosine can escape from degradation by uptake into erythrocytes [6] explaining the observation that the dipeptide can be detected in the urine of volunteers up to 5 hours after oral ingestion [18]. In addition, there are several reports of therapeutic effects of orally ingested carnosine that point towards a delivery of intact carnosine especially to the brain [19-21].

Given the fact that LA-LH had an anti-neoplastic effect comparable to carnosine, being able to discriminate between tumor and non-tumor cells, the other question is whether this compound or other imidazole-containing dipeptides could be a useful alternative to carnosine. This question cannot be answered yet, but it is interesting to note, that our experiments demonstrate that LA-LH is intracellularly more rapidly degraded to its amino acid constituents than carnosine (Figure 3). Therefore, future experiments should investigate whether the release of L-histidine results in a more rapid loss of the bioactive imidazolyl moiety in the cells and whether more stable L-histidine-containing dipeptides could be an alternative to carnosine or LA-LH. More complex synthetic compounds derived from imidazole have already been discussed as potential anti-cancer drugs (for review see [22]). In fact, some of the more complex imidazole derived compounds have already entered the clinics with regard to a number of diseases (for review see [23]), but one has to take into account that synthetic drugs require intensive testing before being used in therapy. On the other hand, carnosine is a naturally occurring compound which has already been used in a number of studies with human patients, and together with its constituent $\beta$-alanine it has a high acceptance as a food supplement for athletes [24]. Imidazole itself has also been discussed as a potential drug for the treatment of colon cancer [25]. But, although Long and Wang used low concentrations in their culture experiments with colon carcinoma cells (up to $36 \mu \mathrm{M}$ ), the high toxicity towards fibroblasts seen in our experiments should be taken into account considering using imidazole as an anti-cancer drug.

Another question addressed by the presented experiments is whether histamine obtained by decarboxylation of L-histidine, could be responsible for carnosine's and L-histidine's anti-neoplastic effect. This notion has been discussed by others [26] and could have been deduced by the fact that histamine had a very strong effect on viability (Figure 1). However, we now rule out this possibility by the observation that we could not detect the formation of histamine after exposure of GBM cells to carnosine, LA-LH or L-histidine. In addition, we also did not detect N-Methylhistamine, a degradation product of histamine, in cells exposed to carnosine, LA-LH or L-histidine, which was detectable in cells exposed to histamine. At this point, it should also be noted, that AOC1 (gene encoding diamine oxidase [EC:1.4.3.22], which is responsible for the conversion of L-histidine to histamine) is almost not present in GBM cells and normal brain tissue (transcripts per million transcripts $\sim 2$ ) as revealed by in silico analysis using data from the TCGA Research Networks (https://www.cancer.gov/tcga; accessed: 22-02-24) and the GTex database https://gtexportal.org/home/; accessed: 22-02-24) using GEPIA (Gene Expression Profiling Interactive Analysis; http://gepia.cancer-pku.cn/; accessed 22-02-24 [27]). In addition, it should be noted that the neuroprotective properties of carnosine are also independent from its metabolization via the L-histidine-histamine pathway [28].

In conclusion, our experiments demonstrate the importance of the L-histidine moiety of carnosine for its anti-neoplastic effect. Although the molecular mechanisms by which this moiety exerts its anti-neoplastic effect have to be revealed in detail, there is evidence that imidazolyl-containing compounds are able to inhibit mitochondrial ATP production [29]. In addition, it has been shown that they can induce cell cycle arrest [30], most likely 
by binding of the imidazolyl moiety to DNA [31]. Despite the observation that the imidazolyl moiety contributes to the anti-neoplastic effect, it is important to note, that we just recently demonstrated that carnosine's influence on tumor cell viability is accompanied by an influence on the pentose phosphate pathway through its interaction with the glycolytic intermediates glyceraldehyde-3-phosphate and dihydroxyacetone phosphate. Therefore, carnosine may have a broader influence on tumor cells than L-histidine alone or other imidazolyl-containing dipeptides [13].

\section{Material and Methods}

Reagents

If not stated otherwise, all chemicals were purchased from Sigma-Aldrich (Taufkirchen, Germany), Merck (Darmstadt, Germany) or Carl Roth (Karlsruhe, Germany). Carnosine was kindly provided by Flamma s.p.a. (Chignolo d'Isola, Italy), L-alanyl-L-histidine and $\beta$-alanyl-L-alanine were purchased from Bachem (Bubendorf, Switzerland).

\section{Cell lines and fibroblast cultures}

The glioblastoma cell line G55T2 was obtained from Sigma (Taufkirchen, Germany), the cell lines U87 and T98G from the ATCC (Manassas, USA) and the line U343 from the German Collection of Microorganisms and Cell Cultures (Braunschweig, Germany). All cells were genotyped (Genolytic $\mathrm{GmbH}$, Leipzig, Germany) and their identity was confirmed.

For cultivation, cells were propagated in $250 \mathrm{~mL}$ culture flasks (Sarstedt AG \& Co., Nümbrecht, Germany) using $10 \mathrm{~mL}$ of standard culture medium (DMEM / $4.5 \mathrm{~g} / \mathrm{L}$ glucose, without pyruvate (Life Technologies, Darmstadt, Germany) supplemented with 10\% fetal bovine serum (FBS superior, Biochrom, Berlin, Germany), 2 mM GlutaMax (Life Technologies) and Penicillin-Streptomycin (Life Technologies)) at $37^{\circ} \mathrm{C}$ and $5 \% \mathrm{CO}_{2}$ in humidified air in an incubator.

Fibroblasts cultures were established as described previously [15]. Briefly, freshly removed galea tissue was washed with PBS (phosphate buffered saline) and minced with a scalpel blade. After mincing, small tissue pieces were transferred to a $25 \mathrm{~cm}^{2}$ culture flask (TPP, Trasadingen, Switzerland) sprinkled with AmnioMax complete medium (Gibco, Darmstadt, Germany). Tissue pieces were incubated for 30 minutes at room temperature and finally, $1 \mathrm{~mL}$ AmnioMax complete medium was added. Incubation was then performed at $37^{\circ} \mathrm{C}, 5 \% \mathrm{CO}_{2}$ and humidified air in an incubator. Medium was changed after 72 hours. As soon as a confluent layer was obtained, cells were removed from culture flasks by use of Accutase (PAA, Pasching, Austria) and transferred to $75 \mathrm{~cm}^{2}$ culture flasks (TPP). AmnioMax Medium with AmnioMax Supplement was used for the first 2-3 weeks of cultivation. Thereafter, fibroblasts were cultivated under the same conditions as glioblastoma cells. Galea tissue was obtained during standard surgery performed at the Neurosurgery Department of the University Hospital Leipzig during 2015 and 2016. All patients provided written informed consent according to the German laws as confirmed by the local committee (144/08-ek).

\section{Cell viability assays}

For cell viability assays, cells were counted and seeded into sterile 96-well plates ( $\mu$ Clear, Greiner Bio One, Frickenhausen, Germany) at a density of 5000 cells/well in 200 $\mu \mathrm{L}$ standard medium. After $24 \mathrm{~h}$ of cultivation $\left(37^{\circ} \mathrm{C}, 5 \% \mathrm{CO}_{2} / 95 \%\right.$ air $)$, the medium was aspirated and fresh medium containing supplements as indicated in each experiment (100 $\mu \mathrm{L} /$ well) and the cells were incubated for additional $48 \mathrm{~h}$. Then, the CellTiter-Glo Luminescent Cell Viability Assay (Promega, Mannheim, Germany) was employed to determine viable cells by measuring ATP in cell lysates and the CellTiter-Blue Cell Viability Assay (Promega) was used to quantify the cell's dehydrogenase activity in living cells. All assays 
were carried out according to manufacturer's protocols. Luminescence and fluorescence were measured using a SpectraMax M5 multilabel reader (Molecular Devices, Biberach, Germany).

\section{Staining and determination of live and dead cells}

The number of live and dead cells after treatment with different compounds was determined in 12-well plates (TPP). Cells were seeded at a density of 80,000 cells per well in $1 \mathrm{~mL}$ of medium. After 24 hours cells received fresh medium with the test compounds. After 48 hours in the presence of the compounds, cells were washed with Hanks balanced salt solution (calcium, magnesium, $1 \mathrm{~g} / \mathrm{L}$ glucose, pH 7.4; Thermo Fisher Scientific, Darmstadt, Germany) before DMEM containing Calcein-AM $(2 \mu \mathrm{M})$, propidium iodide $(1.5 \mu \mathrm{M})$ and Hoechst $33343(2 \mu \mathrm{M})$ was added for 1 hour. Microscopic pictures were taken using a BZ-X800 microscope (Keyence, Neu-Isenburg, Germany) using phase contrast and fluorescence to identify nuclei $(360 / 460 \mathrm{~nm})$, living cells $(470 / 525 \mathrm{~nm})$ and dead cells $(560 / 630$ $\mathrm{nm}$ ). For the determination of the number of nuclei and of dead cells, 9 images (at $4 \mathrm{x}$ magnification) from each well were taken, and ImageJ was used to determine the number of dead cells and nuclei [32].

\section{Determination of intracellular L-histidine}

Intracellular amounts of histidine and histamine were determined as described previously [13]. Briefly, cells were seeded at a density of 300,000 cells per well into a 6-well-plate in $2 \mathrm{~mL}$ of culture medium. After 24-hour cultivation, culture medium was removed and replaced with fresh medium containing specific compounds for each experiment and cells were incubated for additional 48 hours. Then, cells were washed thrice with $1 \mathrm{~mL}$ of ice-cold washing buffer followed by extraction by addition of $400 \mu \mathrm{L}$ of ice-cold methanol. After 10 minutes of gentle shaking on ice, extracts were collected in 1.5 $\mathrm{mL}$ Eppendorf-tubes and wells were rinsed twice in $400 \mu \mathrm{L}$ distilled high quality water (Milli-Q). Samples were evaporated to dryness by lyophilization (Martin Christ Gefriertrocknungsanlagen, Osterode, Germany). For derivatization, the freeze-dried extracts were redissolved in $100 \mu \mathrm{L}$ high quality water (Milli-Q) and $100 \mu \mathrm{L} 0.5 \%$ ortho-phthalaldehyde (dissolved in methanol) were added. Derivatization was carried out at $37^{\circ} \mathrm{C}$ in a thermomixer for 45 minutes, followed by the addition of $800 \mu \mathrm{L} 0.1 \%$ formic acid in HPLC grade water. The obtained solution $(200 \mu \mathrm{L})$ was transferred into $250 \mu \mathrm{L}$ conic glass inserts of $2 \mathrm{~mL}$ ND10 vials, followed by High Performance Liquid Chromatography coupled to Mass Spectrometry (HPLC-MS). After extraction, protein of the remaining layer of fixed

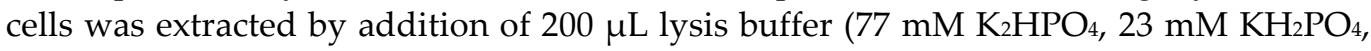
$0.2 \%$ TritonX-100, pH 7.8). Then, total protein was determined by using the Pierce $660 \mathrm{~nm}$ Protein Assay (Thermo Scientific, Braunschweig, Germany).

\section{HPLC-MS set up and data analysis}

An Agilent 1100 series HPLC consisting of a variable wave length detector, a well plate auto sampler and a binary pump, coupled with a Bruker Esquire 3000 plus electrospray ionization mass spectrometer was used. The column was a Phenomenex Gemini $5 \mu \mathrm{C} 18110 \AA 150 \mathrm{~mm} \times 2 \mathrm{~mm}$ with a precolumn. The eluent system consisted of two solvents, with eluent $\mathrm{A}$ : $0.1 \%$ formic acid in acetonitrile and eluent B: $0.1 \%$ formic acid in HPLC grade water. Mobile phase flow rate was $0.5 \mathrm{~mL} / \mathrm{min}$ with the following gradient for separation: $0-10 \mathrm{~min} 90 \% \mathrm{~B}, 90 \%$ to $0 \% \mathrm{~B}$ within $15 \mathrm{~min}, 25-35 \mathrm{~min} 0 \% \mathrm{~B}, 0 \%$ to $90 \% \mathrm{~B}$ within $5 \mathrm{~min}$ and $40-47 \mathrm{~min} 90 \% \mathrm{~B}$ for column equilibration. Mass spectrometer operated in positive mode (target mass: $m / z$ 300; mass range: $m / z 70-400$ ) and dry gas temperature was set to $360^{\circ} \mathrm{C}$ (flow rate: $11 \mathrm{~L} / \mathrm{min} ; 70 \mathrm{psi}$ ). Data was analyzed using OpenChrom version 2.0.103.v20150204-1700 [33]. Histidine and histamine were identified by standards and target masses $\mathrm{m} / \mathrm{z} 272$ (histidine) and 228 (histamine) were used for quantification. If not stated otherwise, the abundance of a metabolite is defined by the peak area 
determined from the selected ion chromatogram of an experiment normalized to the total cellular protein $(\mu \mathrm{g})$. It should be noted that this method doesn't allow the discrimination between D and L stereoisomers. Thus, when referring to signals obtained by HPLC-MS only histidine and not L-histidine is mentioned.

\section{Statistical analysis and graphical representation}

Statistical analysis was carried out using SPSS (IBM, Armonk, USA; Version: 28.0.0.0 (190)). For multiple comparisons, a one-way ANOVA after testing for normality of distribution (Kolmogorov-Smirnov test) was employed, using a Games-Howell or Bonferroni post hoc test after testing for equality of variances (Levene's test). Results were considered to be statistically significant at a value of $\mathrm{p}<0.05$. Graphical representations were prepared using OriginPro (2021b; OriginLab Corporation, Northampton, MA, USA) and CorelDraw Graphics Suite 2020 (Corel Corporation, Ottawa, Canada).

\section{Supplementary Materials}

Supplement 1: Viability of cells from four glioblastoma and from five fibroblasts cultures derived from patients, cultivated in the presence of imidazole-containing compounds

Supplement 2: Microscopic analysis of fibroblast cell cultures and glioblastoma cells in the presence of different compounds.

Funding: This research received no external funding.

Acknowledgments: LC-MS analyses were carried out at the mass spectrometry core facility of the faculty of chemistry and mineralogy at Leipzig University, MS-UL; we thank Dr. Ing. Susan Billig for technical assistance. We like to thank Flamma [Flamma s.p.a. Chignolo d'Isola, Italy (http://www.flammagroup.com)] for the generous supply with very high-quality carnosine for all of our experiments. In addition, we like to thank Dr. Hans-Heinrich Foerster from the Genolytic $\mathrm{GmbH}$ (Leipzig, Germany) for genotyping and confirmation of cell identity.

Author Contributions: Conceptualization, H.O. and F.G.; Methodology, H.O., C.B. and F.G.; Formal Analysis, H.O., C.B. C.S. and F.G.; Investigation, H.O., C.B., C.S., RBS and F.G.; Resources, F.G. and J.M.; Data Curation, H.O. and F.G.; Writing - Original Draft Preparation, H.O., C.S. and F.G.; Writing - Review \& Editing, H.O., C.B., C.S., J.M. and F.G.; Visualization, H.O., C.S. and F.G.; Supervision, H.O. and F.G.; Project Administration, F.G. and J.M.

Institutional Review Board Statement:The study was conducted according to the guidelines of the Declaration of Helsinki, and approved by the Ethics Committee of the Medical Faculty of the University of Leipzig, \#144/08-ek).

Informed Consent Statement: Informed consent was obtained from all subjects involved in the study.

Data Availability Statement: All data of this study is presented in the manuscript and its supplements.

Conflicts of Interest: The authors declare that they have no potential conflict of interest.

\section{References}

1. Ostrom, Q.T.; Patil, N.; Cioffi, G.; Waite, K.; Kruchko, C.; Barnholtz-Sloan, J.S. CBTRUS Statistical Report: Primary Brain and Other Central Nervous System Tumors Diagnosed in the United States in 2013-2017. Neuro-Oncology 2020, 22, iv1-iv96, doi:10.1093/neuonc/noaa200.

2. Hegi, M.E.; Diserens, A.-C.; Gorlia, T.; Hamou, M.-F.; Tribolet, N. de; Weller, M.; Kros, J.M.; Hainfellner, J.A.; Mason, W.; Mariani, L.; et al. MGMT gene silencing and benefit from temozolomide in glioblastoma. N. Engl. J. Med. 2005, 352, 997-1003, doi:10.1056/NEJMoa043331. 
3. Gulewitsch, W.; Amiradzibi, S. Ueber das Carnosin, eine neue organische Base des Fleischextraktes. Ber Dtsch Chem Ges 1900, 33, 1902-1903.

4. Turner, M.D.; Sale, C.; Garner, A.C.; Hipkiss, A.R. Anti-cancer actions of carnosine and the restoration of normal cellular homeostasis. Biochim. Biophys. Acta Mol. Cell Res. 2021, 1868, 119117, doi:10.1016/j.bbamcr.2021.119117.

5. Hipkiss, A.R.; Gaunitz, F. Inhibition of tumour cell growth by carnosine: some possible mechanisms. Amino Acids 2014, 46, 327-337.

6. Oppermann, H.; Elsel, S.; Birkemeyer, C.; Meixensberger, J.; Gaunitz, F. Erythrocytes Prevent Degradation of Carnosine by Human Serum Carnosinase. Int J Mol Sci 2021, 22, doi:10.3390/ijms222312802.

7. Letzien, U.; Oppermann, H.; Meixensberger, J.; Gaunitz, F. The antineoplastic effect of carnosine is accompanied by induction of PDK4 and can be mimicked by L-histidine. Amino Acids 2014, 1009-1019, doi:10.1007/s00726-014-1664-8.

8. Nagai, K.; Suda, T. Antineoplastic effects of carnosine and beta-alanine--physiological considerations of its antineoplastic effects. J Physiol Soc Jpn 1986, 48, 741-747.

9. Shen, Y.; Yang, J.; Li, J.; Shi, X.; Ouyang, L.; Tian, Y.; Lu, J. Carnosine inhibits the proliferation of human gastric cancer SGC-7901 cells through both of the mitochondrial respiration and glycolysis pathways. PLoS ONE 2014, 9, e104632, doi:10.1371/journal.pone.0104632.

10. Iovine, B.; Iannella, M.L.; Nocella, F.; Pricolo, M.R.; Baldi, M.R.; Bevilacqua, M.A. Carnosine inhibits KRas-mediated HCT-116 proliferation by affecting ATP and ROS production. Cancer Lett 2012, 315, 122-128, doi:10.1016/j.canlet.2011.07.021.

11. Ditte, Z.; Ditte, P.; Labudova, M.; Simko, V.; Iuliano, F.; Zatovicova, M.; Csaderova, L.; Pastorekova, S.; Pastorek, J. Carnosine inhibits carbonic anhydrase IX-mediated extracellular acidosis and suppresses growth of HeLa tumor xenografts. BMC Cancer 2014, 14, 358, doi:10.1186/1471-2407-14-358.

12. Renner, C.; Seyffarth, A.; Arriba, S. de; Meixensberger, J.; Gebhardt, R.; Gaunitz, F. Carnosine Inhibits Growth of Cells Isolated from Human Glioblastoma Multiforme. Int J Pept Res Ther 2008, 14, 127-135, doi:10.1007/s10989-007-9121-0.

13. Oppermann, H.; Purcz, K.; Birkemeyer, C.; Baran-Schmidt, R.; Meixensberger, J.; Gaunitz, F. Carnosine's inhibitory effect on glioblastoma cell growth is independent of its cleavage. Amino Acids 2019, 761-772, doi:10.1007/s00726-019-02713-6.

14. Holliday, R.; McFarland, G.A. Inhibition of the growth of transformed and neoplastic cells by the dipeptide carnosine. Br J Cancer 1996, 73, 966-971.

15. Oppermann, H.; Dietterle, J.; Purcz, K.; Morawski, M.; Eisenlöffel, C.; Müller, W.; Meixensberger, J.; Gaunitz, F. Carnosine selectively inhibits migration of IDH-wildtype glioblastoma cells in a co-culture model with fibroblasts. Cancer Cell Int. 2018, 18, 111, doi:10.1186/s12935-018-0611-2.

16. Rauen, U.; Klempt, S.; Groot, H. de. Histidine-induced injury to cultured liver cells, effects of histidine derivatives and of iron chelators. Cell Mol Life Sci 2007, 64, 192-205, doi:10.1007/s00018-006-6456-1.

17. Qiu, J.; Hauske, S.J.; Zhang, S.; Rodriguez-Niño, A.; Albrecht, T.; Pastene, D.O.; van den Born, Jacob; van Goor, H.; Ruf, S.; Kohlmann, M.; et al. Identification and characterisation of carnostatine (SAN9812), a potent and selective carnosinase (CN1) inhibitor with in vivo activity. Amino Acids 2019, 51, 7-16, doi:10.1007/s00726-018-2601-z. 
18. Gardner, M.L.G.; Illingworth, K.M.; Kelleher, J.; Wood, D. Intestinal-Absorption of the Intact Peptide Carnosine in Man, and Comparison with Intestinal Permeability to Lactulose. J Physiol 1991, 439, 411422.

19. Baraniuk, J.N.; El-Amin, S.; Corey, R.; Rayhan, R.; Timbol, C. Carnosine Treatment for Gulf War Illness: A Randomized Controlled Trial. GJHS 2013, 5, doi:10.5539/gjhs.v5n3p69.

20. Hajizadeh-Zaker, R.; Ghajar, A.; Mesgarpour, B.; Afarideh, M.; Mohammadi, M.-R.; Akhondzadeh, S. 1-Carnosine As an Adjunctive Therapy to Risperidone in Children with Autistic Disorder: A Randomized, Double-Blind, Placebo-Controlled Trial. J. Child Adolesc. Psychopharmacol. 2018, 28, 74-81, doi:10.1089/cap.2017.0026.

21. Mehrazad-Saber, Z.; Kheirouri, S.; Noorazar, S.-G. Effects of 1-Carnosine Supplementation on Sleep Disorders and Disease Severity in Autistic Children: A Randomized, Controlled Clinical Trial. Basic Clin. Pharmacol. Toxicol. 2018, 123, 72-77, doi:10.1111/bcpt.12979.

22. Akhtar, J.; Khan, A.A.; Ali, Z.; Haider, R.; Shahar Yar, M. Structure-activity relationship (SAR) study and design strategies of nitrogen-containing heterocyclic moieties for their anticancer activities. Eur J Med Chem 2017, 125, 143-189, doi:10.1016/j.ejmech.2016.09.023.

23. Siwach, A.; Verma, P.K. Synthesis and therapeutic potential of imidazole containing compounds. BMC Chem. 2021, 15, 12, doi:10.1186/s13065-020-00730-1.

24. Saunders, B.; Elliott-Sale, K.; Artioli, G.G.; Swinton, P.A.; Dolan, E.; Roschel, H.; Sale, C.; Gualano, B. $\beta$ alanine supplementation to improve exercise capacity and performance: A systematic review and meta-analysis. Br. J. Sports Med. 2017, 51, 658-669, doi:10.1136/bjsports-2016-096396.

25. Long, Y.; Wang, D. Inhibition of Colon Cancer Cell Growth by Imidazole Through Activation of Apoptotic Pathway. Med. Sci. Monit. 2019, 25, 7597-7604, doi:10.12659/MSM.917779.

26. Zhang, L.; Yao, K.; Fan, Y.; He, P.; Wang, X.; Hu, W.; Chen, Z. Carnosine protects brain microvascular endothelial cells against rotenone-induced oxidative stress injury through histamine $\mathrm{H}_{1}$ and $\mathrm{H}_{2}$ receptors in vitro. Clin. Exp. Pharmacol. Physiol. 2012, 39, 1019-1025, doi:10.1111/1440-1681.12019.

27. Tang, Z.; Li, C.; Kang, B.; Gao, G.; Li, C.; Zhang, Z. GEPIA: a web server for cancer and normal gene expression profiling and interactive analyses. Nucleic Acids Research 2017, 45, W98-W102, doi:10.1093/nar/gkx247.

28. Bae, O.-N.; Majid, A. Role of histidine/histamine in carnosine-induced neuroprotection during ischemic brain damage. Brain Res 2013, 1527, 246-254, doi:10.1016/j.brainres.2013.07.004.

29. Shi, Y.; Lim, S.K.; Liang, Q.; Iyer, S.V.; Wang, H.-Y.; Wang, Z.; Xie, X.; Sun, D.; Chen, Y.-J.; Tabar, V.; et al. Gboxin is an oxidative phosphorylation inhibitor that targets glioblastoma. Nature 2019, 567, 341346, doi:10.1038/s41586-019-0993-x.

30. Morelli, M.B.; Amantini, C.; Nabissi, M.; Cardinali, C.; Santoni, M.; Bernardini, G.; Santoni, A.; Santoni, G. Axitinib induces senescence-associated cell death and necrosis in glioma cell lines: The proteasome inhibitor, bortezomib, potentiates axitinib-induced cytotoxicity in a p21(Waf/Cip1) dependent manner. Oncotarget 2017, 8, 3380-3395, doi:10.18632/oncotarget.13769.

31. Uzlikova, M.; Nohynkova, E. The effect of metronidazole on the cell cycle and DNA in metronidazolesusceptible and -resistant Giardia cell lines. Mol. Biochem. Parasitol. 2014, 198, 75-81, doi:10.1016/j.molbiopara.2015.01.005. 
32. Schneider, C.A.; Rasband, W.S.; Eliceiri, K.W. NIH Image to ImageJ: 25 years of image analysis. Nat. Methods 2012, 9, 671-675.

33. Wenig, P.; Odermatt, J. OpenChrom: a cross-platform open source software for the mass spectrometric analysis of chromatographic data. BMC Bioinformatics 2010, 11, 405, doi:10.1186/1471-2105-11-405. 